\title{
CAMBIOS EN LA TOLERANCIA AL EJERCICIO, CALIDAD DE VIDA Y DISNEA EN PACIENTES CON ENFERMEDAD RESPIRATORIA CRÓNICA QUE ASISTEN A UN PROGRAMA DE REHABILITACIÓN PULMONAR
}

Leslie Vargas-Ramírez ${ }^{1}$, Diana Cano ${ }^{1}$, Fabio Bolívar ${ }^{1}$, Lizeth Rodríguez Corredor ${ }^{1}$, Katherine Rincón ${ }^{1}$, Ingrid Ardila ${ }^{1}$, Mariana Villamizar ${ }^{2}$, Nicolás Farelo

1. Instituto Neumológico del Oriente, 2. Universidad Industrial de Santander. Bucaramanga, Colombia.

OBJETIVO: Evaluar en pacientes con ERC los cambios en la escala de disnea, calidad de vida y tolerancia al ejercicio después del programa de RP del Instituto Neumológico del Oriente (INO).

MATERIALES Y MÉTODOS: Estudio observacional analítico de cohorte retrospectiva en $>18$ años que finalizaron las 48 sesiones de RP del INO. Cambios en test de caminata de seis minutos (TC6M), disnea (mMRC) y calidad de vida (St. George). Para el análisis bivariado pruebas exacta de Fisher y wilcoxon.

RESULTADOS: Inclusión de 75 pacientes, mediana de edad 73 años, 49,3\% mujeres. El diagnostico principal fue EPOC $(62,7 \%)$. Hubo mejoría significativa en las dimensiones de impacto, actividad, síntomas y total de la calidad de vida y disminución en la disnea tras finalizar el programa $(p<0.01)$. No hubo cambios estadísticamente significativos en la tolerancia al ejercicio (TC6M) (Tabla 1).

Tabla 1. Cambio en la mediana del puntaje de calidad de vida y metros del test de caminata de 6 minutos en la población de estudio.

\begin{tabular}{|c|c|c|c|c|}
\hline Variable & $\begin{array}{c}\text { Inicial } \\
\text { Mediana (RI) }\end{array}$ & $\begin{array}{c}\text { Final } \\
\text { Mediana (RI) }\end{array}$ & $\begin{array}{c}\text { Delta } \\
\text { Mediana (RI) }\end{array}$ & Valor $\mathrm{p}^{2}$ \\
\hline Marcha 6 minutos ${ }^{1}$ & $433(392-476)$ & $441(370-476)$ & $3(-25 ; 23)$ & 0,978 \\
\hline \multicolumn{5}{|c|}{ Cuestionario de Saint George } \\
\hline SG Impacto & $23,2(16,5-29,6)$ & $15,3(9,7-23,6)$ & $-5,39(-12,3 ; 0)$ & $<0,01$ \\
\hline SG actividad & $24,9(24,4-25,8)$ & $24,4(18,7-25,0)$ & $-0,18(-1,2 ; 0)$ & $<0,01$ \\
\hline SG síntomas & $44,8(33,0-55,6)$ & $26,1(13,4-49,3)$ & $-13,3(-25,1 ; 0)$ & $<0,01$ \\
\hline SG total & $27,4(21,6-31,7)$ & $20,5(13,9-27,3)$ & $-5,6(-10,8 ;-1,9)$ & $<0,01$ \\
\hline
\end{tabular}

1: metros de distancia recorrida; 2 : test de Wilcoxon

Tabla 2. Cambios en la escala de disnea mMRC en la población de estudio

\begin{tabular}{cccccccc}
$\begin{array}{c}\text { mMRC } \\
\text { inicial }\end{array}$ & $\mathbf{0}$ & $\mathbf{1}$ & $\mathbf{2}$ & $\mathbf{3}$ & $\mathbf{4}$ & Total & Valor $\mathbf{p}^{\mathbf{1}}$ \\
\hline $\mathbf{0}$ & - & - & - & $1(100,0)$ & - & $1(100,0)$ & \\
$\mathbf{1}$ & $6(33,3)$ & $10(55,6)$ & $2(11,1)$ & - & - & $18(100,0)$ & \\
$\mathbf{2}$ & $2(8,0)$ & $15(60,0)$ & $6(24,0)$ & $2(8,0)$ & $0(0,0)$ & $25(100,0)$ & $<0.01$ \\
$\mathbf{3}$ & $3(10,7)$ & $6(21,4)$ & $7(25,0)$ & $8(28,6)$ & $4(14,3)$ & $28(100,09$ & \\
$\mathbf{4}$ & - & - & $1(33,3)$ & $1(33,3)$ & $1(33,3)$ & $3(100,0)$ & \\
\hline Total & $\mathbf{1 1 ( 1 4 , 7 )}$ & $\mathbf{3 1 ( 4 1 , 3 )}$ & $\mathbf{1 6 ( 2 1 , 3 )}$ & $\mathbf{1 2 ( 1 6 , 0 )}$ & $\mathbf{5 ( 6 , 7 )}$ & $\mathbf{7 5 ( 1 0 0 , 0 )}$ & \\
\hline
\end{tabular}

1: Test exacto de Fisher

CONCLUSIONES: Al finalizar el programa de RP del INO hubo mejoria en la disnea y en la calidad de vida, en todas las dimensiones, en pacientes con ERC 